\title{
ON POSITIVENESS AND CONTRACTIVENESS OF THE INTEGRAL OPERATOR ARISING FROM THE BEAM DEFLECTION PROBLEM ON ELASTIC FOUNDATION
}

\author{
Sung Woo Choi
}

\begin{abstract}
We provide a complete proof that there are no eigenvalues of the integral operator $\mathcal{K}_{l}$ outside the interval $(0,1 / k) . \mathcal{K}_{l}$ arises naturally from the deflection problem of a beam with length $2 l$ resting horizontally on an elastic foundation with spring constant $k$, while some vertical load is applied to the beam.
\end{abstract}

\section{Introduction}

We consider the vertical deflection $u(x)$ of a linear-shaped beam with length $2 l>0$ resting horizontally on an elastic foundation. The beam is subject to the downward load distribution $w(x)$ applied vertically on the beam. The given elastic foundation follows Hooke's law with spring constant $k>0$, so that $k \cdot u(x)$ is the spring force distribution by the elastic foundation. Let the constants $E$ and $I$ be Young's modulus and the mass moment of inertia of the beam respectively, so that $E I$ is the flexural rigidity of the beam. According to the classical Euler beam theory, the resulting deflection $u(x)$ is a solution of the following fourth-order linear ODE:

$$
E I \frac{d^{4} u(x)}{d x^{4}}+k \cdot u(x)=w(x) .
$$

The beam deflection problem described above has been one of the cornerstones of mechanical engineering $[1,2,6,8,9,10,11,12,13,14]$. In fact, when the length of the beam is infinite, (1) with the boundary condition $\lim _{x \rightarrow \pm \infty} u(x)=\lim _{x \rightarrow \pm \infty} u^{\prime}(x)=0$ has the following closed form solution [7]:

$$
u(x)=\int_{-\infty}^{\infty} K(|x-\xi|) w(\xi) d \xi .
$$

Received August 21, 2014.

2010 Mathematics Subject Classification. Primary 34L15, 47G10, 74K10.

Key words and phrases. beam, deflection, elastic foundation, integral operator, eigenvalue, $L^{2}$-norm

This work was supported by Duksung Women's University 2013 Research Grant. 
Here, the kernel function $K(\cdot)$ is

$$
K(y):=\frac{\alpha}{2 k} \exp \left(-\frac{\alpha}{\sqrt{2}} y\right) \sin \left(\frac{\alpha}{\sqrt{2}} y+\frac{\pi}{4}\right),
$$

where $\alpha:=\sqrt[4]{k /(E I)}$. By analyzing the integral operator $\mathcal{K}$ defined by

$$
\mathcal{K}[u](x):=\int_{-\infty}^{\infty} K(|x-\xi|) u(\xi) d \xi,
$$

Choi et al. [5] obtained an existence and uniqueness result for the solution of the following nonlinear and nonuniform generalization of (1) for infinitely long beam:

$$
E I \frac{d^{4} u(x)}{d x^{4}}+\phi(u(x), x)=w(x)
$$

To deal with the more practical problem of the nonlinear and nonuniform beam deflection with a finite length $2 l>0$, it is important to analyze the integral operator $\mathcal{K}_{l}$ defined by

$$
\mathcal{K}_{l}[u](x):=\int_{-l}^{l} K(|x-\xi|) u(\xi) d \xi .
$$

Recently, Choi $[3,4]$ performed analysis on the eigenstructure of $\mathcal{K}_{l}$ as a linear operator on the Hilbert space $L^{2}[-l, l]$ of the square-integrable complex functions on $[-l, l]$.

Proposition 1 ([4]). The eigenvalues of $\mathcal{K}_{l}$ inside the real interval $(0,1 / k)$ are $\mu_{1} / k>\nu_{1} / k>\mu_{2} / k>\nu_{2} / k>\cdots \searrow 0$, and $\mu_{n} \sim \nu_{n} \sim n^{-4}$ as $n \rightarrow \infty$.

Since the operator $\mathcal{K}_{l}$ is self-adjoint, all of its eigenvalues are real. In fact, it is shown in [3] that 0 and $1 / k$ are not eigenvalues of $\mathcal{K}_{l}$. About the eigenvalues of $\mathcal{K}_{l}$ in $(-\infty, 0) \cup(1 / k, \infty)$, they obtained a characteristic equation in terms of specific functions $\psi_{L}(\kappa)$ and $q(\kappa)$ defined in Section 2 .

Proposition $2([3]) . \lambda \in(-\infty, 0) \cup(1 / k, \infty)$ is an eigenvalue of $\mathcal{K}_{l}$ if and only if $\psi_{L}(\kappa)=q(\kappa)$, where $\kappa=\sqrt[4]{1-1 /(\lambda k)}>0$ and $L=2 \sqrt{2} l \alpha$.

In this paper, we provide a complete proof of the fact

$$
\psi_{L}(\kappa)>q(\kappa) \text { for every } \kappa>0 \text { and for every } L>0,
$$

from which the following result follows immediately by Proposition 2 .

Theorem 1. There are no eigenvalues of the operator $\mathcal{K}_{l}$ outside the interval $(0,1 / k)$.

Theorem 1 implies that the operator $\mathcal{K}_{l}$ is positive and contractive in dimension-free sense, which is relevant to the existence and the uniqueness of the solution to the nonlinear and nonuniform problem (2). We remark that the proof of Lemma 3.2 in [3], which also asserts (3), was incomplete in that it only amounts to showing that $\psi_{L}(\kappa)>q(\kappa)$ for every sufficiently small $\kappa>0$ 
for every $L>0$, which is indeed far from complete. However, our proof of (3) indicates that the conclusions of [3], including Lemma 3.2, Theorems 4.1 and 4.2 therein, remain unchanged.

\section{Preliminaries}

For $\kappa \geq 0$, define

$$
\begin{aligned}
q(\kappa) & =\frac{(\kappa-1)^{2}}{(\kappa+1)^{2}}, \\
\psi_{L}(\kappa) & =e^{L \kappa} \cdot f\left(\cos g_{L}(\kappa)\right),
\end{aligned}
$$

where

$$
f(t)=(2-t)-\sqrt{(2-t)^{2}-1} .
$$

Here, $L:=2 \sqrt{2} l \alpha, l, \alpha$ are positive constants, and the function $g_{L}$, parametrized by $L>0$, is one-to-one and onto from $[0, \infty)$ to $[0, \infty)$ with $g_{L}(0)=0$. Specifically, $g_{L}$, which was denoted by $g$ in [3], is defined as follows:

$$
g_{L}(\kappa)=L \kappa-\hat{g}(\kappa)
$$

where

$$
\hat{g}(\kappa)= \begin{cases}\arctan \left\{\frac{4 \kappa\left(\kappa^{2}-1\right)}{\kappa^{4}-6 \kappa^{2}+1}\right\} & \text { if } 0 \leq \kappa<\sqrt{2}-1, \\ -\frac{\pi}{2} & \text { if } \kappa=\sqrt{2}-1, \\ -\pi+\arctan \left\{\frac{4 \kappa\left(\kappa^{2}-1\right)}{\kappa^{4}-6 \kappa^{2}+1}\right\} & \text { if } \sqrt{2}-1<\kappa<\sqrt{2}+1, \\ -\frac{3 \pi}{2} & \text { if } \kappa=\sqrt{2}+1, \\ -2 \pi+\arctan \left\{\frac{4 \kappa\left(\kappa^{2}-1\right)}{\kappa^{4}-6 \kappa^{2}+1}\right\} & \text { if } \kappa>\sqrt{2}+1 .\end{cases}
$$

Here, the branch of $\arctan$ is taken such that $\arctan (0)=0$. As is shown in [3], $\hat{g}$ is continuous and differentiable on $[0, \infty)$, and is strictly decreasing from $\hat{g}(0)=0$ to $\lim _{\kappa \rightarrow \infty} \hat{g}(\kappa)=-2 \pi$. In fact, we have [3, pp. 43-44]

$$
\begin{gathered}
\hat{g}^{\prime}(\kappa)=-\frac{4}{\kappa^{2}+1}, \\
g_{L}{ }^{\prime}(\kappa)=L+\frac{4}{\kappa^{2}+1} .
\end{gathered}
$$

The inverse $g_{L}^{-1}$ of $g_{L}$ is differentiable, and is one-to-one and onto from $[0, \infty)$ to $[0, \infty)$ with $g_{L}^{-1}(0)=0$.

Note that the function $q$ is differentiable. The function $\psi_{L}$ is continuous, but is only piecewise differentiable. (See Lemma 2(a) and its proof below.) The following observation, which is immediate from the intermediate value theorem and the mean value theorem, plays a key role in our proof of (3), and hence Theorem 1. 
Proposition 3. Suppose $\xi$ and $\eta$ are continuous and piecewise differentiable functions on $[a, b]$ satisfying $\xi(a) \geq \eta(a)$ and $\xi(b) \geq \eta(b)$, and possible discontinuities of $\xi^{\prime}$ and $\eta^{\prime}$ are discrete. Suppose the equation $\xi(\kappa) \leq \eta(\kappa)$ has a solution in $(a, b)$, and $\xi$ and $\eta$ are differentiable at every such solution. Then there exists $\kappa_{0}$ in $(a, b)$ such that $\xi\left(\kappa_{0}\right) \leq \eta\left(\kappa_{0}\right)$ and $\xi^{\prime}\left(\kappa_{0}\right)=\eta^{\prime}\left(\kappa_{0}\right)$.

\section{The functions $\psi_{L}$ and $q$}

We first examine properties of the functions $\psi_{L}$ and $q$. From (4), we have

$$
\begin{aligned}
q^{\prime}(\kappa) & =\left\{\frac{(\kappa-1)^{2}}{(\kappa+1)^{2}}\right\}^{\prime}=\frac{2(\kappa-1) \cdot(\kappa+1)^{2}-(\kappa-1)^{2} \cdot 2(\kappa+1)}{(\kappa+1)^{4}} \\
& =\frac{2(\kappa-1)\{(\kappa+1)-(\kappa-1)\}}{(\kappa+1)^{3}}=\frac{4(\kappa-1)}{(\kappa+1)^{3}} .
\end{aligned}
$$

The properties of the function $q(\kappa)$ that we need, are summarized in Lemma 1 , whose proof is immediate from (4) and (11).

Lemma 1. $q$ is strictly decreasing on $[0,1]$ from $q(0)=1$ to $q(1)=0$, and strictly increasing on $[1, \infty)$ approaching 1 . In particular, $0 \leq q(\kappa)<1$ for $\kappa>0$.

Note that the function $f$ in $(6)$ is continuous and positive. It is differentiable except at $t=1$. In fact, we have

(12) $f^{\prime}(t)=-1-\frac{2(2-t) \cdot(-1)}{2 \sqrt{(2-t)^{2}-1}}=-1+\frac{2-t}{\sqrt{(2-t)^{2}-1}}=\frac{f(t)}{\sqrt{(2-t)^{2}-1}}$

$$
\geq 0
$$

and hence $f$ is increasing. It follows that

$$
0<3-2 \sqrt{2} \leq f\left(\cos g_{L}(\kappa)\right) \leq 1 \quad \text { for } \kappa>0,
$$

since $-1 \leq \cos g_{L}(\kappa) \leq 1$ and $f(-1)=3-2 \sqrt{2}, f(1)=1$. So $\psi_{L}(\kappa)=$ $e^{L \kappa} f(\cos \kappa) \geq(3-2 \sqrt{2}) e^{L \kappa}$, and hence we have

$$
\begin{aligned}
& \psi_{L}(\kappa)>0 \text { for } \kappa>0, L>0, \\
& \lim _{\kappa \rightarrow \infty} \psi_{L}(\kappa)=\infty \quad \text { for } L>0 .
\end{aligned}
$$

By (12), we have

$$
\begin{aligned}
\psi_{L}^{\prime}(\kappa) & =e^{L \kappa}\left\{L \cdot f\left(\cos g_{L}(\kappa)\right)+f^{\prime}\left(\cos g_{L}(\kappa)\right) \cdot\left(-\sin g_{L}(\kappa)\right) \cdot g_{L}{ }^{\prime}(\kappa)\right\} \\
& =e^{L \kappa}\left[L \cdot f\left(\cos g_{L}(\kappa)\right)+\frac{f\left(\cos g_{L}(\kappa)\right) \cdot\left(-\sin g_{L}(\kappa)\right) \cdot g_{L}{ }^{\prime}(\kappa)}{\sqrt{\left(2-\cos g_{L}(\kappa)\right)^{2}-1}}\right] \\
& =\psi_{L}(\kappa)\left\{L-\frac{\sin g_{L}(\kappa)}{\left.\sqrt{\left(2-\cos g_{L}(\kappa)\right)^{2}-1} \cdot g_{L}{ }^{\prime}(\kappa)\right\} .}\right.
\end{aligned}
$$


Using the identity

$$
(2-\cos t)^{2}-1=\cos ^{2} t-4 \cos t+3=(1-\cos t)(3-\cos t),
$$

we have

$$
\begin{aligned}
\lim _{t \rightarrow 0 \pm} \frac{\sin t}{\sqrt{(2-\cos t)^{2}-1}} & =\lim _{t \rightarrow 0 \pm} \frac{ \pm \sqrt{(1-\cos t)(1+\cos t)}}{\sqrt{(1-\cos t)(3-\cos t)}} \\
& = \pm \lim _{t \rightarrow 0 \pm} \frac{\sqrt{(1+\cos t)}}{\sqrt{(3-\cos t)}}= \pm 1
\end{aligned}
$$

Since

$$
\begin{aligned}
\left(\frac{\sin t}{\sqrt{(2-\cos t)^{2}-1}}\right)^{\prime} & =\frac{\cos t \cdot \sqrt{(2-\cos t)^{2}-1}-\sin t \cdot \frac{2(2-\cos t) \cdot \sin t}{2 \sqrt{(2-\cos t)^{2}-1}}}{(2-\cos t)^{2}-1} \\
& =\frac{\cos t \cdot\left\{(2-\cos t)^{2}-1\right\}-\left(1-\cos ^{2} t\right)(2-\cos t)}{{\sqrt{(2-\cos t)^{2}-1}}^{3}} \\
& =\frac{-2 \cos ^{2} t+4 \cos t-2}{{\sqrt{(2-\cos t)^{2}-1}}^{3}}=-\frac{2(1-\cos t)^{2}}{{\sqrt{(2-\cos t)^{2}-1}}^{3}} \leq 0
\end{aligned}
$$

the periodic function $\sin t / \sqrt{(2-\cos t)^{2}-1}$ is strictly decreasing on $(0,2 \pi)$, and hence, together with (18), we have

$$
-1 \leq \frac{\sin t}{\sqrt{(2-\cos t)^{2}-1}} \leq 1 .
$$

Lemma 2. (a) $\psi_{L}$ is differentiable at every $\kappa>0$ such that $\psi_{L}(\kappa) \leq q(\kappa)$.

(b) $\psi_{L}{ }^{\prime}(\kappa) \geq-\psi_{L}(\kappa) \cdot 4 /\left(\kappa^{2}+1\right)$ for every $\kappa>0$ where $\psi_{L}$ is differentiable.

Proof. Let $\kappa>0$. By $(16), \psi_{L}$ is differentiable except at $g_{L}^{-1}(2 \pi n)$ for $n=$ $1,2,3, \ldots$ For $n=1,2,3, \ldots, \psi_{L}\left(g_{L}^{-1}(2 \pi n)\right)=e^{L \cdot g_{L}^{-1}(2 \pi n)} \cdot f(2 \pi n)=e^{L \cdot g_{L}^{-1}(2 \pi n)}$ $>1$ by $(5)$ and $(6)$, and $q\left(g_{L}^{-1}(2 \pi n)\right)<1$ by Lemma 1 . So $\psi_{L}\left(g_{L}^{-1}(2 \pi n)\right)>$ $q\left(g_{L}^{-1}(2 \pi n)\right)$ for $n=1,2,3, \ldots$, which shows (a).

By $(16),(19)$, we have $\psi_{L}^{\prime}(\kappa) \geq \psi_{L}(\kappa) \cdot\left\{L-g_{L}{ }^{\prime}(\kappa)\right\}$, since $\psi_{L}(\kappa)>0$ by (14) and $g_{L}{ }^{\prime}(\kappa)>0$ by (10). Hence (b) follows from (10).

\section{Proof of the main result}

In proving (3), we will divide the cases into the following: (i) When $0<\kappa \leq$ 1 , and (ii) when $\kappa>1$. The former case is settled with Lemma 3 below.

Lemma 3. If $0<\kappa \leq 1$, then $\psi_{L}(\kappa)>q(\kappa)$ for every $L>0$. 
Proof. Note first that $\psi_{L}(1)>0=q(1)$ by (4) and (14). So (3) holds when $\kappa=1$. Note also that $\psi_{L}(0)=1=q(0)$ by (4) and (5). Suppose (3) is not true for $0<\kappa<1$, so that there exists a solution of the equation $\psi_{L}(\kappa) \leq q(\kappa)$ in $(0,1)$ for some $L>0$. By Lemma $2(\mathrm{a}), \psi_{L}$ and $q$ are differentiable at every such solution. Thus we can apply Proposition 3 to $\psi_{L}$ and $q$ on $[0,1]$, so that there exists $\kappa_{0}$ in $(0,1)$ satisfying $\psi_{L}\left(\kappa_{0}\right) \leq q\left(\kappa_{0}\right), \psi_{L}^{\prime}\left(\kappa_{0}\right)=q^{\prime}\left(\kappa_{0}\right)$. So by (14) and Lemma 2(b), we have

$$
q^{\prime}\left(\kappa_{0}\right)=\psi_{L}^{\prime}\left(\kappa_{0}\right) \geq-\psi_{L}\left(\kappa_{0}\right) \cdot \frac{4}{\kappa_{0}^{2}+1} \geq-q\left(\kappa_{0}\right) \cdot \frac{4}{\kappa_{0}^{2}+1},
$$

and hence by (4) and (11),

$$
\frac{4\left(\kappa_{0}-1\right)}{\left(\kappa_{0}+1\right)^{3}} \geq-\frac{\left(\kappa_{0}-1\right)^{2}}{\left(\kappa_{0}+1\right)^{2}} \cdot \frac{4}{\kappa_{0}^{2}+1} .
$$

Since $0<\kappa_{0}<1$, this is equivalent to $\kappa_{0}^{2}+1 \leq-\left(\kappa_{0}^{2}-1\right)$, or $\kappa_{0}^{2} \leq 0$, which implies $\kappa_{0}=0$. This is a contradiction, and so we conclude $\psi_{L}(\kappa)>q(\kappa)$ for every $0<\kappa \leq 1$.

For the rest of the paper, we will deal with the case $\kappa>1$. The next result shows the nature of the equation $\psi_{L}(\kappa) \leq q(\kappa)$ with respect to $L$.

Lemma 4. Suppose the equation $\psi_{L_{0}}(\kappa) \leq q(\kappa)$ has a positive solution for some $L_{0}>0$. Then, for each $L$ with $0<L \leq L_{0}$, there exists $\kappa_{L}>1$ such that $\psi_{L}\left(\kappa_{L}\right) \leq q\left(\kappa_{L}\right)$ and $\psi_{L}^{\prime}\left(\kappa_{L}\right)=q^{\prime}\left(\kappa_{L}\right)$.

Proof. Suppose the equation $\psi_{L_{0}}(\kappa) \leq q(\kappa)$ has a solution $\kappa_{0}>0$ for some $L_{0}>0$. Note that $\kappa_{0}>1$ by Lemma 3. From (7), we have $\partial g_{L}(\kappa) / \partial L=\kappa$. So from (5) and (12), we have

$$
\begin{aligned}
\frac{\partial \psi_{L}(\kappa)}{\partial L} & =\frac{\partial}{\partial L}\left\{e^{L \kappa} \cdot f\left(\cos g_{L}(\kappa)\right)\right\} \\
& =\kappa e^{L \kappa} \cdot f\left(\cos g_{L}(\kappa)\right)+e^{L \kappa} \cdot f^{\prime}\left(\cos g_{L}(\kappa)\right) \cdot\left(-\sin g_{L}(\kappa)\right) \cdot \frac{\partial g_{L}(\kappa)}{\partial L} \\
& =\kappa \cdot e^{L \kappa} f\left(\cos g_{L}(\kappa)\right)-e^{L \kappa} \cdot \frac{f\left(\cos g_{L}(\kappa)\right) \sin g_{L}(\kappa)}{\sqrt{\left(2-\cos g_{L}(\kappa)\right)^{2}-1}} \cdot \kappa \\
& =\kappa \cdot \psi_{L}(\kappa)\left\{1-\frac{\sin g_{L}(\kappa)}{\sqrt{\left(2-\cos g_{L}(\kappa)\right)^{2}-1}}\right\} \geq 0,
\end{aligned}
$$

where we used (14) and (19) for the last inequality. Thus $\psi_{L}\left(\kappa_{0}\right)$ is increasing with respect to $L$, and hence $\psi_{L}\left(\kappa_{0}\right) \leq \psi_{L_{0}}\left(\kappa_{0}\right) \leq q\left(\kappa_{0}\right)$ for every $L$ such that $0<L<L_{0}$.

Note that $\psi_{L}(1)>0=q(1)$ for every $L>0$. Since $\lim _{\kappa \rightarrow \infty} q(\kappa)=1$ by Lemma 1 and $\lim _{\kappa \rightarrow \infty} \psi_{L}(\kappa)=\infty$ by $(15)$, there exists $b_{L}>x_{0}>1$ such that $\psi_{L}\left(b_{L}\right)>q\left(b_{L}\right)$ for each $L>0$. By Lemma $2(\mathrm{a}), \psi_{L}$ and $q$ are differentiable at every $\kappa \in\left(1, b_{L}\right)$ such that $\psi_{L}(\kappa) \leq q(\kappa)$. Thus, for each $L$ 
such that $0<L<L_{0}$, we can apply Proposition 3 to $\psi_{L}$ and $q$ on $\left[1, b_{L}\right]$, so that there exists $\kappa_{L} \in\left(1, b_{L}\right) \subset(1, \infty)$ satisfying $\psi_{L}\left(\kappa_{L}\right) \leq q\left(\kappa_{L}\right)$ and $\psi_{L}{ }^{\prime}\left(\kappa_{L}\right)=q^{\prime}\left(\kappa_{L}\right)$.

Lemma 5. Suppose $\psi_{L}(\kappa) \leq q(\kappa)$ for some $\kappa>0$ and $L>0$. Then $\kappa>$ $1+\sqrt{2}$.

Proof. For $L>0$, the condition $\psi_{L}(\kappa) \leq q(\kappa)$ implies

$$
\frac{(\kappa-1)^{2}}{(\kappa+1)^{2}} \geq e^{L \kappa} f\left(\cos g_{L}(\kappa)\right) \geq e^{L \kappa}(3-2 \sqrt{2})>3-2 \sqrt{2}
$$

by (4), (5), (13), and hence

$$
\begin{aligned}
0 & <(\kappa-1)^{2}-(3-2 \sqrt{2})(\kappa+1)^{2} \\
& =(2 \sqrt{2}-2) \kappa^{2}-2(4-2 \sqrt{2}) \kappa+(2 \sqrt{2}-2) \\
& =(2 \sqrt{2}-2)\left\{\kappa^{2}-2 \sqrt{2} \kappa+1\right\} \\
& =(2 \sqrt{2}-2)\{\kappa-(\sqrt{2}-1)\}\{\kappa-(\sqrt{2}+1)\} .
\end{aligned}
$$

So we have $\kappa<\sqrt{2}-1$ or $\kappa>\sqrt{2}+1$. It follows that $\kappa>\sqrt{2}+1$, since $\kappa>1$ by Lemma 3 .

In view of Lemma 4 , it is legitimate to consider the behavior of (hypothetical) $\kappa_{L}$, as $L \searrow 0$.

Lemma 6. Suppose $\psi_{L}\left(\kappa_{L}\right) \leq q\left(\kappa_{L}\right)$ and $\psi_{L}{ }^{\prime}\left(\kappa_{L}\right)=q^{\prime}\left(\kappa_{L}\right)$ with $\kappa_{L}>0$. Then $\lim _{L \rightarrow 0+} \kappa_{L}=\infty$.

Proof. Note first that $\kappa_{L}>1$ by Lemma 3. From the assumption $\psi_{L}{ }^{\prime}\left(\kappa_{L}\right)=$ $q^{\prime}\left(\kappa_{L}\right)$ and (16), we have

$$
q^{\prime}\left(\kappa_{L}\right)=\psi_{L}{ }^{\prime}\left(\kappa_{L}\right)=\psi_{L}\left(\kappa_{L}\right)\left\{L-\frac{\sin g_{L}\left(\kappa_{L}\right)}{\sqrt{\left(2-\cos g_{L}\left(\kappa_{L}\right)\right)^{2}-1}} \cdot g_{L}{ }^{\prime}\left(\kappa_{L}\right)\right\} .
$$

Since $q^{\prime}\left(\kappa_{L}\right)>0$ by (11) and $\psi_{L}\left(\kappa_{L}\right)>0$ by (14), we have

$$
L-\frac{\sin g_{L}\left(\kappa_{L}\right)}{\sqrt{\left(2-\cos g_{L}\left(\kappa_{L}\right)\right)^{2}-1}} \cdot g_{L}{ }^{\prime}\left(\kappa_{L}\right)>0,
$$

and hence

$$
q^{\prime}\left(\kappa_{L}\right) \leq q\left(\kappa_{L}\right)\left\{L-\frac{\sin g_{L}\left(\kappa_{L}\right)}{\sqrt{\left(2-\cos g_{L}\left(\kappa_{L}\right)\right)^{2}-1}} \cdot g_{L}{ }^{\prime}\left(\kappa_{L}\right)\right\}
$$


by the assumption $\psi_{L}\left(\kappa_{L}\right) \leq q\left(\kappa_{L}\right)$. So by (4), (11), we have

$$
\frac{4}{\kappa_{L}^{2}-1}=\frac{q^{\prime}\left(\kappa_{L}\right)}{q\left(\kappa_{L}\right)} \leq L-\frac{\sin g_{L}\left(\kappa_{L}\right)}{\sqrt{\left(2-\cos g_{L}\left(\kappa_{L}\right)\right)^{2}-1}} \cdot g_{L}{ }^{\prime}\left(\kappa_{L}\right),
$$

and hence

$$
g_{L}{ }^{\prime}\left(\kappa_{L}\right) \sin g_{L}\left(\kappa_{L}\right) \leq\left(L-\frac{4}{\kappa_{L}^{2}-1}\right) \sqrt{\left(2-\cos g_{L}\left(\kappa_{L}\right)\right)^{2}-1} .
$$

If $L-\frac{4}{\kappa_{L}^{2}-1} \geq 0$, which is equivalent to $\kappa_{L} \geq \sqrt{1+\frac{4}{L}}$, then $\lim _{L \rightarrow 0+} \kappa_{L} \geq$ $\lim _{L \rightarrow 0+} \sqrt{1+\frac{4}{L}}=\infty$, and hence we have $\lim _{L \rightarrow 0+} \kappa_{L}=\infty$. So we assume $L-\frac{4}{\kappa^{2}-1}<0$ for the rest of the proof. Then the right side, and hence the left side as well, of (20) becomes negative. By squaring the both nonnegative sides of

$$
-g_{L}{ }^{\prime}\left(\kappa_{L}\right) \sin g_{L}\left(\kappa_{L}\right) \geq-\left(L-\frac{4}{\kappa_{L}^{2}-1}\right) \sqrt{\left(2-\cos g_{L}\left(\kappa_{L}\right)\right)^{2}-1},
$$

we have

$$
\begin{aligned}
& \left\{g_{L}{ }^{\prime}\left(\kappa_{L}\right)\right\}^{2}\left(1-\cos ^{2} g_{L}\left(\kappa_{L}\right)\right) \\
\geq & \left(L-\frac{4}{\kappa_{L}^{2}-1}\right)^{2}\left\{\left(2-\cos g_{L}\left(\kappa_{L}\right)\right)^{2}-1\right\} \\
= & \left(L-\frac{4}{\kappa_{L}^{2}-1}\right)^{2}\left\{\cos ^{2} g_{L}\left(\kappa_{L}\right)-4 \cos g_{L}\left(\kappa_{L}\right)+3\right\},
\end{aligned}
$$

and hence

$$
\begin{aligned}
0 \geq & \left\{\left\{g_{L}{ }^{\prime}\left(\kappa_{L}\right)\right\}^{2}+\left(L-\frac{4}{\kappa_{L}^{2}-1}\right)^{2}\right\} \cos ^{2} g_{L}\left(\kappa_{L}\right) \\
& -4\left(L-\frac{4}{\kappa_{L}^{2}-1}\right)^{2} \cos g_{L}\left(\kappa_{L}\right)+\left\{3\left(L-\frac{4}{\kappa_{L}^{2}-1}\right)^{2}-\left\{g_{L}{ }^{\prime}\left(\kappa_{L}\right)\right\}^{2}\right\} .
\end{aligned}
$$

So we have $\alpha \leq \cos g_{L}\left(\kappa_{L}\right) \leq \beta$, where $\alpha, \beta$ are (interchangeably)

$$
\begin{aligned}
& \frac{1}{\left\{g_{L}{ }^{\prime}\left(\kappa_{L}\right)\right\}^{2}+\left(L-\frac{4}{\kappa_{L}^{2}-1}\right)^{2}}\left[2\left(L-\frac{4}{\kappa_{L}^{2}-1}\right)^{2} \pm\left\{4\left(L-\frac{4}{\kappa_{L}^{2}-1}\right)^{4}\right.\right. \\
& \left.\left.-\left\{\left\{g_{L}{ }^{\prime}\left(\kappa_{L}\right)\right\}^{2}+\left(L-\frac{4}{\kappa_{L}^{2}-1}\right)^{2}\right\}\left\{3\left(L-\frac{4}{\kappa_{L}^{2}-1}\right)^{2}-\left\{g_{L}{ }^{\prime}\left(\kappa_{L}\right)\right\}^{2}\right\}\right\}^{\frac{1}{2}}\right] \\
& =\frac{2\left(L-\frac{4}{\kappa_{L}^{2}-1}\right)^{2} \pm\left|\left\{g_{L}{ }^{\prime}\left(\kappa_{L}\right)\right\}^{2}-\left(L-\frac{4}{\kappa_{L}^{2}-1}\right)^{2}\right|}{\left\{g_{L}{ }^{\prime}\left(\kappa_{L}\right)\right\}^{2}+\left(L-\frac{4}{\kappa_{L}^{2}-1}\right)^{2}}
\end{aligned}
$$




$$
=1, \quad \frac{-\left\{g_{L}{ }^{\prime}\left(\kappa_{L}\right)\right\}^{2}+3\left(L-\frac{4}{\kappa_{L}^{2}-1}\right)^{2}}{\left\{g_{L}{ }^{\prime}\left(\kappa_{L}\right)\right\}^{2}+\left(L-\frac{4}{\kappa_{L}^{2}-1}\right)^{2}} .
$$

Note that $\cos g_{L}\left(\kappa_{L}\right)<1$ by Lemma $2(\mathrm{a})$ and its proof. Thus we must have

$$
\frac{-\left\{g_{L}{ }^{\prime}\left(\kappa_{L}\right)\right\}^{2}+3\left(L-\frac{4}{\kappa_{L}^{2}-1}\right)^{2}}{\left\{g_{L}{ }^{\prime}\left(\kappa_{L}\right)\right\}^{2}+\left(L-\frac{4}{\kappa_{L}^{2}-1}\right)^{2}}<1,
$$

which is equivalent to

$$
\left(L-\frac{4}{\kappa_{L}^{2}-1}\right)^{2}<\left\{g_{L}{ }^{\prime}\left(\kappa_{L}\right)\right\}^{2}=\left(L+\frac{4}{\kappa_{L}^{2}+1}\right)^{2}
$$

by (10). Since we assumed that $L-4 /\left(\kappa_{L}^{2}-1\right)<0$, we have

and hence

$$
-\left(L-\frac{4}{\kappa_{L}^{2}-1}\right)<L+\frac{4}{\kappa_{L}^{2}+1}
$$

$$
L>\frac{1}{2}\left(\frac{4}{\kappa_{L}^{2}-1}-\frac{4}{\kappa_{L}^{2}+1}\right)=\frac{4}{\kappa_{L}^{4}-1}
$$

which is equivalent to $\kappa_{L}>\sqrt[4]{1+\frac{4}{L}}$. So $\lim _{L \rightarrow 0+} \kappa_{L} \geq \lim _{L \rightarrow 0+} \sqrt[4]{1+\frac{4}{L}}=\infty$. Thus we have $\lim _{L \rightarrow 0+} \kappa_{L}=\infty$, and the proof is complete.

Lemma 7. Suppose $\psi_{L}\left(\kappa_{L}\right) \leq q\left(\kappa_{L}\right)$ and $\psi_{L}^{\prime}\left(\kappa_{L}\right)=q^{\prime}\left(\kappa_{L}\right)$ with $\kappa_{L}>0$. Then $g_{L}\left(\kappa_{L}\right)<2 \pi$ and $\lim _{L \rightarrow 0+} g_{L}\left(\kappa_{L}\right)=2 \pi$.

Proof. From the assumption $\psi_{L}\left(\kappa_{L}\right)=e^{L \kappa_{L}} \cdot f\left(\cos g_{L}\left(\kappa_{L}\right)\right) \leq q\left(\kappa_{L}\right)$, we have

$$
\begin{aligned}
\frac{e^{L \kappa_{L}}}{q\left(\kappa_{L}\right)} & \leq \frac{1}{f\left(\cos g_{L}\left(\kappa_{L}\right)\right)}=\frac{1}{2-\cos g_{L}\left(\kappa_{L}\right)-\sqrt{\left(2-\cos g_{L}\left(\kappa_{L}\right)\right)^{2}-1}} \\
& =2-\cos g_{L}\left(\kappa_{L}\right)+\sqrt{\left(2-\cos g_{L}\left(\kappa_{L}\right)\right)^{2}-1}
\end{aligned}
$$

Since $\cos t=\cos (t-2 \pi) \geq 1-(t-2 \pi)^{2} / 2$, we have $2-\cos t \leq 2-\left\{1-(t-2 \pi)^{2} / 2\right\}$ $=1+(t-2 \pi)^{2} / 2$, and hence

$$
\begin{aligned}
2-\cos t+\sqrt{(2-\cos t)^{2}-1} & \leq 1+\frac{(t-2 \pi)^{2}}{2}+\sqrt{\left\{1+\frac{(t-2 \pi)^{2}}{2}\right\}^{2}-1} \\
& =1+\frac{(t-2 \pi)^{2}}{2}+\sqrt{(t-2 \pi)^{2}+\frac{(t-2 \pi)^{4}}{4}} \\
& =1+\frac{(t-2 \pi)^{2}}{2}+|t-2 \pi| \sqrt{1+\frac{(t-2 \pi)^{2}}{4}} \\
& \leq 1+\frac{(t-2 \pi)^{2}}{2}+|t-2 \pi|\left\{1+\frac{(t-2 \pi)^{2}}{8}\right\}
\end{aligned}
$$




$$
=1+|t-2 \pi|+\frac{|t-2 \pi|^{2}}{2}+\frac{|t-2 \pi|^{3}}{8}
$$

for every $t \in \mathbb{R}$, where we used the inequality $\sqrt{1+x^{2} / 4} \leq 1+x^{2} / 8$ for the second inequality. So we have

$$
\frac{e^{L \kappa_{L}}}{q\left(\kappa_{L}\right)} \leq 1+\left|g_{L}\left(\kappa_{L}\right)-2 \pi\right|+\frac{1}{2}\left|g_{L}\left(\kappa_{L}\right)-2 \pi\right|^{2}+\frac{1}{8}\left|g_{L}\left(\kappa_{L}\right)-2 \pi\right|^{3} .
$$

Note that, since $\kappa_{L}>1+\sqrt{2}$ by Lemma 5 ,

$$
g_{L}\left(\kappa_{L}\right)-2 \pi=L \kappa_{L}-\hat{g}\left(\kappa_{L}\right)-2 \pi=L \kappa_{L}-\arctan \frac{4 \kappa_{L}\left(\kappa_{L}^{2}-1\right)}{\kappa_{L}^{4}-6 \kappa_{L}^{2}+1}
$$

by (7) and (8). So from the inequality $e^{x}>1+x+\frac{x^{2}}{2}+\frac{x^{3}}{6}$ for $x>0$, we have

$$
\begin{aligned}
& \frac{1}{q\left(\kappa_{L}\right)}\left\{1+L \kappa_{L}+\frac{1}{2}\left(L \kappa_{L}\right)^{2}+\frac{1}{6}\left(L \kappa_{L}\right)^{3}\right\} \\
< & 1+\left|L \kappa_{L}-\arctan \frac{4 \kappa_{L}\left(\kappa_{L}^{2}-1\right)}{\kappa_{L}^{4}-6 \kappa_{L}^{2}+1}\right|+\frac{1}{2}\left|L \kappa_{L}-\arctan \frac{4 \kappa_{L}\left(\kappa_{L}^{2}-1\right)}{\kappa_{L}^{4}-6 \kappa_{L}^{2}+1}\right|^{2} \\
+ & \frac{1}{8}\left|L \kappa_{L}-\arctan \frac{4 \kappa_{L}\left(\kappa_{L}^{2}-1\right)}{\kappa_{L}^{4}-6 \kappa_{L}^{2}+1}\right|^{3},
\end{aligned}
$$

or equivalently,

$$
\begin{aligned}
& \quad 24+24 L \kappa_{L}+12\left(L \kappa_{L}\right)^{2}+4\left(L \kappa_{L}\right)^{3} \\
& <24 q\left(\kappa_{L}\right)+24 q\left(\kappa_{L}\right)\left|L \kappa_{L}-\arctan \frac{4 \kappa_{L}\left(\kappa_{L}^{2}-1\right)}{\kappa_{L}^{4}-6 \kappa_{L}^{2}+1}\right| \\
& +12 q\left(\kappa_{L}\right)\left|L \kappa_{L}-\arctan \frac{4 \kappa_{L}\left(\kappa_{L}^{2}-1\right)}{\kappa_{L}^{4}-6 \kappa_{L}^{2}+1}\right|^{2} \\
& +3 q\left(\kappa_{L}\right)\left|L \kappa_{L}-\arctan \frac{4 \kappa_{L}\left(\kappa_{L}^{2}-1\right)}{\kappa_{L}^{4}-6 \kappa_{L}^{2}+1}\right|^{3} .
\end{aligned}
$$

Suppose

$$
L \kappa_{L} \geq \arctan \frac{4 \kappa_{L}\left(\kappa_{L}^{2}-1\right)}{\kappa_{L}^{4}-6 \kappa_{L}^{2}+1}
$$

Then (22) becomes

$$
\begin{aligned}
0> & \left\{4-3 q\left(\kappa_{L}\right)\right\}\left(L \kappa_{L}\right)^{3} \\
& +\left\{12+9 q\left(\kappa_{L}\right) \arctan \frac{4 \kappa_{L}\left(\kappa_{L}^{2}-1\right)}{\kappa_{L}^{4}-6 \kappa_{L}^{2}+1}-12 q\left(\kappa_{L}\right)\right\}\left(L \kappa_{L}\right)^{2} \\
& +\left\{24-9 q\left(\kappa_{L}\right) \arctan ^{2} \frac{4 \kappa_{L}\left(\kappa_{L}^{2}-1\right)}{\kappa_{L}^{4}-6 \kappa_{L}^{2}+1}\right.
\end{aligned}
$$




$$
\begin{gathered}
\left.+24 q\left(\kappa_{L}\right) \arctan \frac{4 \kappa_{L}\left(\kappa_{L}^{2}-1\right)}{\kappa_{L}^{4}-6 \kappa_{L}^{2}+1}-24 q\left(\kappa_{L}\right)\right\} L \kappa_{L} \\
+\left\{24+3 q\left(\kappa_{L}\right) \arctan ^{3} \frac{4 \kappa_{L}\left(\kappa_{L}^{2}-1\right)}{\kappa_{L}^{4}-6 \kappa_{L}^{2}+1}-12 q\left(\kappa_{L}\right) \arctan ^{2} \frac{4 \kappa_{L}\left(\kappa_{L}^{2}-1\right)}{\kappa_{L}^{4}-6 \kappa_{L}^{2}+1}\right. \\
\left.+24 q\left(\kappa_{L}\right) \arctan \frac{4 \kappa_{L}\left(\kappa_{L}^{2}-1\right)}{\kappa_{L}^{4}-6 \kappa_{L}^{2}+1}-24 q\left(\kappa_{L}\right)\right\}
\end{gathered}
$$

and hence

$$
\left(L \kappa_{L}\right)^{3}+a\left(L \kappa_{L}\right)^{2}+b L \kappa_{L}+c<0,
$$

where

$$
\begin{aligned}
& a= \frac{12\left\{1-q\left(\kappa_{L}\right)\right\}}{4-3 q\left(\kappa_{L}\right)}+\frac{9 q\left(\kappa_{L}\right)}{4-3 q\left(\kappa_{L}\right)} \arctan \frac{4 \kappa_{L}\left(\kappa_{L}^{2}-1\right)}{\kappa_{L}^{4}-6 \kappa_{L}^{2}+1}, \\
& b=\frac{24\left\{1-q\left(\kappa_{L}\right)\right\}}{4-3 q\left(\kappa_{L}\right)}-\frac{q\left(\kappa_{L}\right)}{4-3 q\left(\kappa_{L}\right)} \arctan \frac{4 \kappa_{L}\left(\kappa_{L}^{2}-1\right)}{\kappa_{L}^{4}-6 \kappa_{L}^{2}+1} \cdot\left\{9 \arctan \frac{4 \kappa_{L}\left(\kappa_{L}^{2}-1\right)}{\kappa_{L}^{4}-6 \kappa_{L}^{2}+1}-24\right\}, \\
& c=\frac{24\left\{1-q\left(\kappa_{L}\right)\right\}}{4-3 q\left(\kappa_{L}\right)}+\frac{3 q\left(\kappa_{L}\right)}{4-3 q\left(\kappa_{L}\right)} \arctan \frac{4 \kappa_{L}\left(\kappa_{L}^{2}-1\right)}{\kappa_{L}^{4}-6 \kappa_{L}^{2}+1} \\
& \quad \cdot\left\{\arctan ^{2} \frac{4 \kappa_{L}\left(\kappa_{L}^{2}-1\right)}{\kappa_{L}^{4}-6 \kappa_{L}^{2}+1}-4 \arctan \frac{4 \kappa_{L}\left(\kappa_{L}^{2}-1\right)}{\kappa_{L}^{4}-6 \kappa_{L}^{2}+1}+8\right\} .
\end{aligned}
$$

Since $\kappa_{L}>1+\sqrt{2}$ and

$$
\begin{aligned}
\kappa^{4}-6 \kappa^{2}+1 & =\left(\kappa^{2}-1\right)^{2}-4 \kappa^{2}=\left(\kappa^{2}+2 \kappa-1\right)\left(\kappa^{2}-2 \kappa-1\right) \\
& =(\kappa+1+\sqrt{2})(\kappa+1-\sqrt{2})(\kappa-1+\sqrt{2})(\kappa-1-\sqrt{2}),
\end{aligned}
$$

we have $4 \kappa_{L}\left(\kappa_{L}^{2}-1\right) /\left(\kappa_{L}^{4}-6 \kappa_{L}^{2}+1\right)>0$, and hence

$$
0<\arctan \frac{4 \kappa_{L}\left(\kappa_{L}^{2}-1\right)}{\kappa_{L}^{4}-6 \kappa_{L}^{2}+1}<\frac{\pi}{2} \approx 1.5708 .
$$

Again since $\kappa_{L}>1+\sqrt{2}$, we have $0<q\left(\kappa_{L}\right)<1$ by Lemma 1 , and hence

$$
\frac{1-q\left(\kappa_{L}\right)}{4-3 q\left(\kappa_{L}\right)}>0, \quad \frac{q\left(\kappa_{L}\right)}{4-3 q\left(\kappa_{L}\right)}>0 .
$$

It follows that $a, b, c>0$, which is a contradiction to (23) since $L \kappa_{L}>0$. Hence we have

$$
L \kappa_{L}<\arctan \frac{4 \kappa_{L}\left(\kappa_{L}^{2}-1\right)}{\kappa_{L}^{4}-6 \kappa_{L}^{2}+1} .
$$


By (21) and (24), we have

$$
g_{L}\left(\kappa_{L}\right)=L \kappa_{L}-\arctan \frac{4 \kappa_{L}\left(\kappa_{L}^{2}-1\right)}{\kappa_{L}^{4}-6 \kappa_{L}^{2}+1}+2 \pi<2 \pi .
$$

Since $L \kappa_{L}>0$, we have

$$
-\arctan \frac{4 \kappa_{L}\left(\kappa_{L}^{2}-1\right)}{\kappa_{L}^{4}-6 \kappa_{L}^{2}+1}<L \kappa_{L}-\arctan \frac{4 \kappa_{L}\left(\kappa_{L}^{2}-1\right)}{\kappa_{L}^{4}-6 \kappa_{L}^{2}+1}<0
$$

by (24). So by Lemma 6 ,

$$
\begin{aligned}
0 & \geq \lim _{L \rightarrow 0+}\left\{L \kappa_{L}-\arctan \frac{4 \kappa_{L}\left(\kappa_{L}^{2}-1\right)}{\kappa_{L}^{4}-6 \kappa_{L}^{2}+1}\right\} \geq-\lim _{L \rightarrow 0+} \arctan \frac{4 \kappa_{L}\left(\kappa_{L}^{2}-1\right)}{\kappa_{L}^{4}-6 \kappa_{L}^{2}+1} \\
& =-\lim _{\kappa_{L} \rightarrow \infty} \arctan \frac{4 \kappa_{L}\left(\kappa_{L}^{2}-1\right)}{\kappa_{L}^{4}-6 \kappa_{L}^{2}+1}=0,
\end{aligned}
$$

and hence we have

$$
\lim _{L \rightarrow 0+}\left\{L \kappa_{L}-\arctan \frac{4 \kappa_{L}\left(\kappa_{L}^{2}-1\right)}{\kappa_{L}^{4}-6 \kappa_{L}^{2}+1}\right\}=0 .
$$

Thus by (21) again, we have

$$
\lim _{L \rightarrow 0+} g_{L}\left(\kappa_{L}\right)=\lim _{L \rightarrow 0+}\left\{L \kappa_{L}-\lim _{L \rightarrow 0+} \arctan \frac{4 \kappa_{L}\left(\kappa_{L}^{2}-1\right)}{\kappa_{L}^{4}-6 \kappa_{L}^{2}+1}\right\}+2 \pi=2 \pi,
$$

which completes the proof.

Lemma 7 indicates that it is enough to consider the case when $g_{L}(\kappa)<2 \pi$ to prove (3). We will do the change of the variables from $\kappa$ to $t$ via $t=g_{L}(\kappa)$ for $\kappa \geq 0$, or equivalently, $\kappa=g_{L}^{-1}(t)$ for $t \geq 0$.

Lemma 8. Suppose $0<t<2 \pi$. Then $\lim _{L \rightarrow 0+} g_{L}^{-1}(t)=\hat{g}^{-1}(-t)$, and $g_{L}^{-1}(t)<\hat{g}^{-1}(-t)$ for every $L>0$.

Proof. From the definition (7) of $g_{L}$, we have

$$
L \cdot g_{L}^{-1}(t)-\hat{g}\left(g_{L}^{-1}(t)\right)=t,
$$

Differentiating with respect to $L$, we have

$$
1 \cdot g_{L}^{-1}(t)+L \cdot \frac{\partial}{\partial L} g_{L}^{-1}(t)-\hat{g}^{\prime}\left(g_{L}^{-1}(t)\right) \cdot \frac{\partial}{\partial L} g_{L}^{-1}(t)=0,
$$

and hence by (7) and (10),

$$
\frac{\partial}{\partial L} g_{L}^{-1}(t)=-\frac{g_{L}^{-1}(t)}{L-\hat{g}^{\prime}\left(g_{L}^{-1}(t)\right)}=-\frac{\kappa}{L-\hat{g}^{\prime}(\kappa)}=-\frac{\kappa}{g_{L}^{\prime}(\kappa)}<0,
$$

where we put $\kappa=g_{L}^{-1}(t)$. This shows that $g_{L}^{-1}(t)$ is strictly decreasing with respect to $L$ for any fixed $t$, and consequently, $g_{L}^{-1}(t)$ is strictly increasing as $L \searrow 0$. 
Suppose $0<t<2 \pi$. If $\lim _{L \rightarrow 0+} g_{L}^{-1}(t)=\infty$, then by (8) and (25), we have

$$
\begin{aligned}
2 \pi>t & =\lim _{L \rightarrow 0+}\left\{L \cdot g_{L}^{-1}(t)\right\}-\lim _{L \rightarrow 0+}\left\{\hat{g}\left(g_{L}^{-1}(t)\right)\right\} \\
& =\lim _{L \rightarrow 0+}\left\{L \cdot g_{L}^{-1}(t)\right\}-\lim _{\kappa \rightarrow \infty}\{\hat{g}(\kappa)\} \\
& =\lim _{L \rightarrow 0+}\left\{L \cdot g_{L}^{-1}(t)\right\}-(-2 \pi) \geq 2 \pi,
\end{aligned}
$$

which is a contradiction. So $\lim _{L \rightarrow 0+} g_{L}^{-1}(t)<\infty$. Note from (25) again that

$$
t=\lim _{L \rightarrow 0+} L \cdot \lim _{L \rightarrow 0+} g_{L}^{-1}(t)-\lim _{L \rightarrow 0+}\left\{\hat{g}\left(g_{L}^{-1}(t)\right)\right\}=0-\hat{g}\left(\lim _{L \rightarrow 0+} g_{L}^{-1}(t)\right),
$$

from which it follows that $\lim _{L \rightarrow 0+} g_{L}^{-1}(t)=\hat{g}^{-1}(-t)$. Since $g_{L}^{-1}(t)$ is strictly decreasing with respect to $L$, we have $g_{L}^{-1}(t)<\hat{g}^{-1}(-t)$ for every $L>0$.

We remark that, in fact, $\lim _{L \rightarrow 0+} g_{L}^{-1}(t)=\infty$ for every $t \geq 2 \pi$, whose proof we omit. For $t \geq 0$, define

$$
\tilde{\psi}_{L}(t)=\psi_{L}\left(g_{L}^{-1}(t)\right), \quad \tilde{q}_{L}(t)=q\left(g_{L}^{-1}(t)\right) .
$$

The functions $\tilde{\psi}_{L}$ and $\tilde{q}_{L}$ can be considered as "mollified" versions of $\psi_{L}$ and $q$ as $L \searrow 0$. From the definitions of $\psi_{L}$ and $\tilde{\psi}_{L}$, we have

$$
\tilde{\psi}_{L}(t)=e^{L \cdot g_{L}^{-1}(t)} f(\cos t)>f(\cos t) \quad \text { for } t>0 .
$$

Note that $\hat{g}^{-1}(-3 \pi / 2)=1+\sqrt{2}$ by $(8)$, and $g_{L}^{-1}(3 \pi / 2)$ is strictly increasing to $\hat{g}^{-1}(-3 \pi / 2)=1+\sqrt{2}$ as $L$ goes down to 0 by Lemma 8 . It follows that, for every sufficiently small $L>0$, we have $g_{L}^{-1}(t)>1$ for $3 \pi / 2<t<2 \pi$. Since $q$ is strictly increasing on $(1, \infty)$ by Lemma 1 , we have

$$
\begin{aligned}
\tilde{q}_{L}(t)=q\left(g_{L}^{-1}(t)\right)<q\left(\hat{g}^{-1}(-t)\right) & \text { for } 3 \pi / 2<t<2 \pi \\
& \text { for every sufficiently small } L>0
\end{aligned}
$$

by Lemma 8 .

Lemma 9. For every sufficiently small $L>0, \tilde{\psi}_{L}(t)>\tilde{q}_{L}(t)$ for $3 \pi / 2<t<$ $2 \pi$.

Proof. By (26) and (27), it is enough to show that $f(\cos t)>q\left(\hat{g}^{-1}(-t)\right)$ for $3 \pi / 2<t<2 \pi$. Suppose $3 \pi / 2<t<2 \pi$. Note that $\kappa:=\hat{g}^{-1}(-t)>1+\sqrt{2}$ by (8). So by (8) again, we have

$$
-t=\hat{g}(\kappa)=-2 \pi+\arctan \frac{4 \kappa\left(\kappa^{2}-1\right)}{\kappa^{4}-6 \kappa^{2}+1},
$$

and hence

$$
\frac{4 \kappa\left(\kappa^{2}-1\right)}{\kappa^{4}-6 \kappa^{2}+1}=\tan (2 \pi-t)=-\tan t
$$


Note that, for each $t \in(3 \pi / 2,2 \pi)$, we have $-\tan t>0$, and $\kappa$ is the unique positive solution of $(28)$ such that $\kappa>1+\sqrt{2}$. Transform (28) to

$$
-\tan t \cdot\left(\kappa^{4}-6 \kappa^{2}+1\right)=4 \kappa\left(\kappa^{2}-1\right),
$$

and then to

$$
4\left(\kappa-\frac{1}{\kappa}\right)=-\tan t \cdot\left(\kappa^{2}-6+\frac{1}{\kappa^{2}}\right)=-\tan t \cdot\left\{\left(\kappa-\frac{1}{\kappa}\right)^{2}-4\right\} .
$$

Putting

$$
x=\kappa-\frac{1}{\kappa}
$$

we have $4 x=-\tan t \cdot\left(x^{2}-4\right)$, and hence $\tan t \cdot x^{2}+4 x-4 \tan t=0$, which gives

$$
x=\frac{-2 \pm \sqrt{4+4 \tan ^{2} t}}{\tan t}=\frac{-2 \cos t \pm 2}{\sin t} .
$$

Note that $\sin t<0$ for $3 \pi / 2<t<2 \pi$. Since $\kappa>1$, we have $x>0$ by (29), and hence

$$
x=\frac{-2 \cos t-2}{\sin t}=\frac{-2(1+\cos t)}{\sin t} .
$$

Substituting (30) into (29) again, we have

$$
\sin t \cdot \kappa^{2}+2(1+\cos t) \kappa-\sin t=0 .
$$

Solving (31) for $\kappa$, we have

$$
\kappa=\frac{-(1+\cos t) \pm \sqrt{(1+\cos t)^{2}+\sin ^{2} t}}{\sin t}=\frac{-(1+\cos t) \pm \sqrt{2} \sqrt{1+\cos t}}{\sin t} .
$$

Since $\kappa>0$ and $\sin t<0$, we finally have

$$
\hat{g}^{-1}(-t)=\kappa=\frac{-(1+\cos t)-\sqrt{2} \sqrt{1+\cos t}}{\sin t}=\frac{\sqrt{1+\cos t}+\sqrt{2}}{\sqrt{1-\cos t}},
$$

and thus by (4),

$$
\begin{aligned}
& q\left(\hat{g}^{-1}(-t)\right) \\
= & \left\{\frac{\frac{\sqrt{1+\cos t}+\sqrt{2}}{\sqrt{1-\cos t}}-1}{\frac{\sqrt{1+\cos t}+\sqrt{2}}{\sqrt{1-\cos t}}+1}\right\}^{2}=\left\{\frac{\sqrt{1+\cos t}+\sqrt{2}-\sqrt{1-\cos t}}{\sqrt{1+\cos t}+\sqrt{2}+\sqrt{1-\cos t}}\right\}^{2} \\
= & \left\{\frac{\sqrt{1+\cos t}+\sqrt{2}-\sqrt{1-\cos t}}{\sqrt{1+\cos t}+\sqrt{2}+\sqrt{1-\cos t}} \cdot \frac{\sqrt{1+\cos t}+\sqrt{2}-\sqrt{1-\cos t}}{\sqrt{1+\cos t}+\sqrt{2}-\sqrt{1-\cos t}}\right\}^{2} \\
= & \frac{1}{\{(1+\cos t)+2 \sqrt{2} \sqrt{1+\cos t}+2-(1-\cos t)\}^{2}} \\
& \cdot\{(1+\cos t)+(1-\cos t)+2+2 \sqrt{2} \sqrt{1+\cos t}
\end{aligned}
$$




$$
\begin{aligned}
& -2 \sqrt{2} \sqrt{1-\cos t}-2 \sqrt{1-\cos t} \sqrt{1+\cos t}\}^{2} \\
= & \left\{\frac{2 \sqrt{2}(\sqrt{1+\cos t}+\sqrt{2})-2 \sqrt{1-\cos t}(\sqrt{1+\cos t}+\sqrt{2})}{2 \sqrt{1+\cos t}(\sqrt{1+\cos t}+\sqrt{2})}\right\}^{2} \\
= & \left\{\frac{\sqrt{2}-\sqrt{1-\cos t}}{\sqrt{1+\cos t}}\right\}^{2}=\frac{3-\cos t-2 \sqrt{2} \sqrt{1-\cos t}}{1+\cos t} .
\end{aligned}
$$

By (6), it remains to show that

$$
2-\cos t-\sqrt{(2-\cos t)^{2}-1}>\frac{3-\cos t-2 \sqrt{2} \sqrt{1-\cos t}}{1+\cos t}
$$

for $3 \pi / 2<t<2 \pi$, which is done by the following series of equivalent transformations:

$$
\begin{gathered}
-\cos ^{2} t+\cos t+2-(1+\cos t) \sqrt{(2-\cos t)^{2}-1}>3-\cos t-2 \sqrt{2} \sqrt{1-\cos t}, \\
(1-\cos t)^{2}+(1+\cos t) \sqrt{(1-\cos t)(3-\cos t)}<2 \sqrt{2} \sqrt{1-\cos t}, \\
\sqrt{1-\cos t}^{3}+(1+\cos t) \sqrt{3-\cos t}<2 \sqrt{2}, \\
(1-\cos t)^{3}<8+(1+\cos t)^{2}(3-\cos t)-4 \sqrt{2}(1+\cos t) \sqrt{3-\cos t}, \\
2 \cos ^{2} t-8 \cos t-10<-4 \sqrt{2}(1+\cos t) \sqrt{3-\cos t}, \\
(1+\cos t)(5-\cos t)>2 \sqrt{2}(1+\cos t) \sqrt{3-\cos t}, \\
\cos ^{2} t-10 \cos t+25>8(3-\cos t), \\
\cos ^{2} t-2 \cos t+1>0,
\end{gathered}
$$

where we used (17) for the second inequality.

We now have all the ingredients needed to prove (3), which implies Theorem 1.

Proof of Theorem 1. By Proposition 2, it is sufficient to show (3). Suppose (3) is false, so that the equation $\psi_{L_{0}}(\kappa) \leq q(\kappa)$ has a positive solution for some $L_{0}>0$. Then by Lemma 4 , there exists $\kappa_{L}$ satisfying $\psi_{L}\left(\kappa_{L}\right) \leq q\left(\kappa_{L}\right)$ and $\psi_{L}{ }^{\prime}\left(\kappa_{L}\right)=q^{\prime}\left(\kappa_{L}\right)$ for $0<L<L_{0}$. Let $t_{N}:=g_{L}\left(\kappa_{L}\right)$ for $0<L<L_{0}$. By Lemma 7 , we have $3 \pi / 2<t_{L}<2 \pi$ for every sufficiently small $L>0$. So by Lemma 9 , we have $\tilde{\psi}_{L}\left(t_{L}\right)>\tilde{q}_{L}\left(t_{L}\right)$, and hence

$$
\psi_{L}\left(\kappa_{L}\right)=\psi_{L}\left(g_{L}^{-1}\left(t_{L}\right)\right)=\tilde{\psi}_{L}\left(t_{L}\right)>\tilde{q}_{L}\left(t_{L}\right)=q\left(g_{L}^{-1}\left(t_{L}\right)\right)=q\left(\kappa_{L}\right)
$$

for every sufficiently small $L>0$. This is a contradiction to the result that $\psi_{L}\left(\kappa_{L}\right) \leq q\left(\kappa_{L}\right)$ for $0<L<L_{0}$. Thus we conclude that (3) is true. 


\section{References}

1] E. Alves, E. A. de Toledo, L. A. P. Gomes, and M. B. de Souza Cortes, A note on iterative solutions for a nonlinear fourth order ode, Bol. Soc. Parana. Mat. (3) 27 (2009), no. 1, $15-20$.

[2] F. W. Beaufait and P. W. Hoadley, Analysis of elastic beams on nonlinear foundations, Comput. Struct. 12 (1980), 669-676.

[3] S. W. Choi, Spectral analysis of the integral operator arising from the beam deflection problem on elastic foundation I: positiveness and contractiveness, J. Appl. Math. Inform. 30 (2012), no. 1-2, 27-47.

[4] Spectral analysis of the integral operator arising from the beam deflection problem on elastic foundation II: eigenvalues, Bound. Value Probl. 2015 (2015), no. 6, 27pp.

[5] S. W. Choi and T. S. Jang, Existence and uniqueness of nonlinear deflections of an infinite beam resting on a non-uniform nonlinear elastic foundation, Bound. Value Probl. 2012 (2012), no. 5, 24 pp.

[6] M. Galewski, On the nonlinear elastic simply supported beam equation, An. Ştiinţ. Univ. "Ovidius" Constanţa Ser. Mat. 19 (2011), no. 1, 109-119.

[7] M. D. Greenberg, Foundations of Applied Mathematics, Prentice-Hall Inc., 1978.

[8] M. R. Grossinho and A. I. Santos, Solvability of an elastic beam equation in presence of a sign-type Nagumo control, Nonlinear Stud. 18 (2011), no. 2, 279-291.

[9] M. Hetenyi, Beams on Elastic Foundation, The University of Michigan Press, Ann Arbor, Mich., 1946.

[10] Y. H. Kuo and S. Y. Lee, Deflection of non-uniform beams resting on a nonlinear elastic foundation, Comput. Struct. 51 (1994), 513-519.

[11] C. Miranda and K. Nair, Finite beams on elastic foundation, ASCE. I. Struct. Div. 92 (1966), 131-142.

[12] S. P. Timoshenko, Statistical and dynamical stress in rails, Proceedings of the International Congress on Applied Mechanics (Zurich), 407-418, 1926

[13] , Strength of Materials: part $1 \&$ part 2, 3rd ed., Van Nostrand, Princeton, NJ, 1955

[14] B. Y. Ting, Finite beams on elastic foundation with restraints, ASCE. J. Struct. Div. 108 (1982), 611-621.

Department of Mathematics

DUKSUNG WOMEN'S UNIVERSITY

SeOul 132-714, KoreA

E-mail address: swchoi@duksung.ac.kr 